\title{
LETTERS
}

\section{Cardiac shunt in COPD as a cause of severe hypoxaemia: probably not so uncommon after all}

\section{To the Editors:}

Hypoxaemia is a common finding in chronic obstructive pulmonary disease (COPD) and may be aggravated during exercise. The main mechanism is perfusion through areas that are not well ventilated: a ventilation-perfusion mismatch. True shunting, defined as venous blood mixing directly with endcapillary blood at the arterial side of the circulation, is not a usual cause of hypoxaemia in COPD [1, 2]. The amount of shunting related to ventilation-perfusion mismatch is usually made by the calculation of the shunt fraction while the patient is inhaling $100 \%$ oxygen. We present two cases of severe hypoxaemia in patients with COPD to show that a cardiac shunt can contribute to hypoxaemia, and that this shunt can be missed if the $100 \%$ oxygen method is used to quantify shunt.

Patient A was a 68-yr-old male, referred from another hospital to our clinic for evaluation of severe dyspnoea and hypoxaemia. Patient complaints were rapidly progressive dyspnoea and severe impaired exercise tolerance with blue discoloration of the fingers and lips for 3 months. There were no complaints of coughing, sputum production or fever. Patient A ceased smoking 12 yrs previously after 60 pack-yrs. Physical examination revealed peripheral and central cyanosis, and a little ankle oedema. Arterial blood gas analysis revealed hypoxaemia at rest, with an arterial oxygen tension $\left(\mathrm{Pa}_{2} \mathrm{O}_{2}\right)$ of $42 \mathrm{mmHg}$ and an arterial oxygen saturation $\left(\mathrm{Sa}_{1} \mathrm{O}_{2}\right)$ of $77 \%$. Pulmonary function tests showed moderate obstruction (forced expiratory volume in $1 \mathrm{~s}$ (FEV1) of $67 \%$ predicted and $\mathrm{FEV} 1 /$ synchronised vital capacity (SVC) ratio of $65 \%$ pred). Initiation of exercise resulted in immediate oxygen desaturation to $71 \%$, preventing any further exercise. Radiological analysis by means of highresolution computed tomography (CT), pulmonary angiography and ventilation-perfusion scan revealed only signs of emphysema. Transthoracic and transoesophageal echocardiography showed dilation of the right atrium and ventricle with signs of pulmonary hypertension. No signs of an intracardiac right-to-left shunt were seen. Both tests were performed while $15 \mathrm{~L} \cdot \mathrm{min}^{-1}$ oxygen was administered. Right heart catheterisation confirmed the diagnosis of pulmonary hypertension (table 1). The response to administration of $100 \%$ oxygen was a fall of mean pulmonary arterial pressure $(\mathrm{Ppa})$ to $24 \mathrm{mmHg}$, accompanied by a significant increase in arterial oxygenation to $96 \%$.

For this reason, we assumed that a right-to-left shunt through a patent foramen ovale (PFO) was present under normoxic conditions and during exercise, although no intracardiac shunt was seen during echocardiography. Cardiac magnetic resonance imaging (MRI) was performed while the patient was breathing room air. Flow per beat was measured in the pulmonary artery $(44 \mathrm{~mL})$ and aorta $(56 \mathrm{~mL})$, which resembled a right-to-left shunt of $25 \%$. We concluded from these measurements that oxygen desaturation during exercise under normoxic conditions occured as a consequence of right-to-left shunting through a PFO. The patient was referred to a cardiologist for a percutaneous closure of the PFO, which resulted in an improved clinical condition together with an oxygen saturation of $95 \%$ at rest which remained unaltered during exercise. Control MRI showed similar flow per beat through the aorta and pulmonary artery (57 mL).

Patient B was a 70-yr-old male with a history of COPD, hypercapnia and echocardiographic signs of pulmonary hypertension $(\mathrm{PH})$. Patient $\mathrm{B}$ complained of progressive dyspnoea on exercise with blue discoloration of lips and fingers for several months. He had experienced no acute exacerbations of his COPD in the previous year. Patient B ceased smoking 18 yrs previously. Arterial blood gas analysis showed hypoxaemia $\left(\mathrm{Pa}_{2} \mathrm{O}_{2} 48 \mathrm{mmHg} ; \mathrm{Sa}_{1} \mathrm{O}_{2} 81 \%\right)$ and hypercapnia (carbon dioxide tension $56 \mathrm{mmHg}$ ). Hypercapnia was interpreted as a sign of respiratory insufficiency for which a trial of bilevel positive airway pressure (BiPAP) treatment was tried in the past, resulting in a further worsening of his

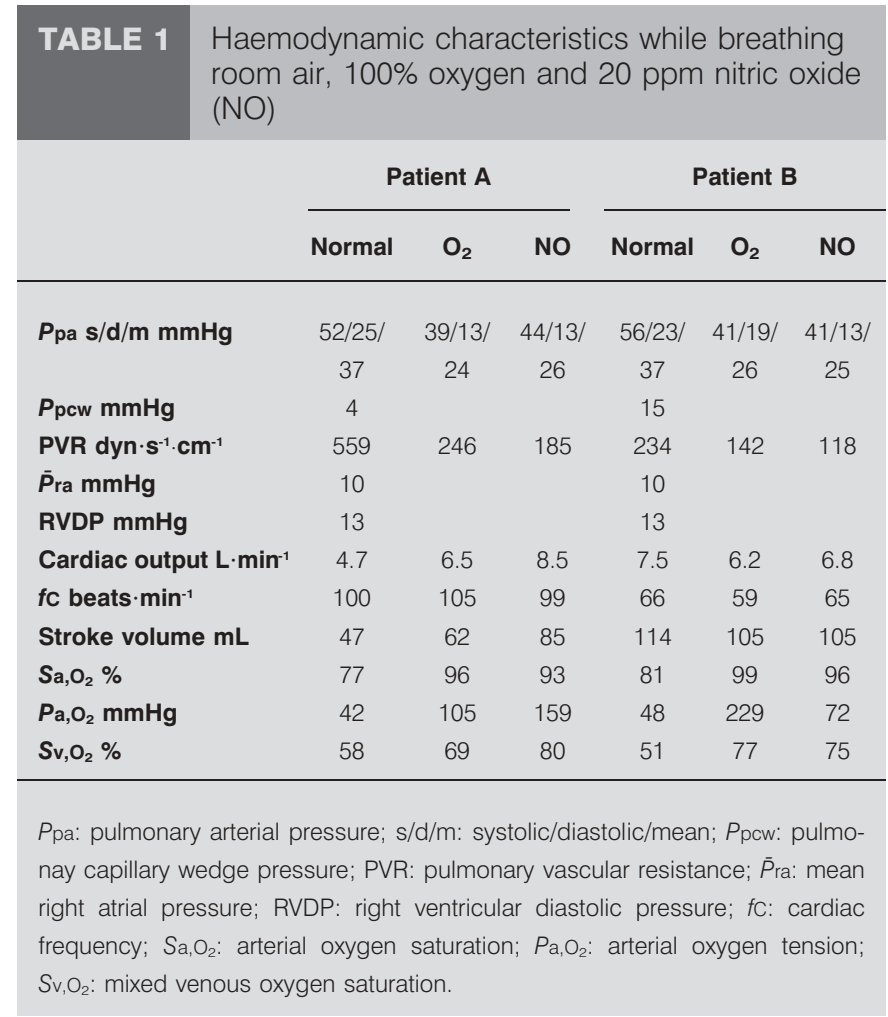


hypercapnia and hypoxaemia. Pulmonary function tests showed severe obstruction (FEV1 41\% pred and FEV1/SVC $37 \%$ pred). During cardiopulmonary exercise testing, patient $B$ reached $70 \mathrm{~W}$ (44\% pred), which led to an oxygen desaturation to $71 \%$ while heart rate reserve was $21 \%$ and ventilatory reserve was $50 \%$. For this reason, hypoxaemia was considered the exercise-limiting factor. High-resolution CT, pulmonary angiography and ventilation-perfusion scan showed severe emphysema without evidence for pulmonary embolisms. Echocardiography, with oxygen $\left(15 \mathrm{~L} \cdot \mathrm{min}^{-1}\right)$, showed dilatation of right atrium and right ventricle, and signs of $\mathrm{PH}$. No intracardiac shunt was found. Right heart catheterisation was performed, which revealed increased $P$ pa and right atrial pressure (Pra) (table 1). Inhalation of $20 \mathrm{ppm} \mathrm{NO}$ led to a normalisation of $P$ pa and oxygen saturation. Based on this finding, it was concluded that the severe desaturation during exercise was most likely due to right-to-left shunting through an PFO in the presence of PH secondary to COPD. Echocardiography was repeated under normoxic conditions, showing a PFO at a location unsuitable for percutaneous closure. Because of the good response to inhaled $\mathrm{NO}$ a trial of a phosphodiesterase (PDE)-5 inhibitor was initiated, leading to clinical improvement and a stable oxygen saturation of $90 \%$. After 4 yrs, patient B was re-evaluated, showing a stable condition with a $\mathrm{Sa}_{2} \mathrm{O}_{2}$ of $91 \%$ at rest with a similar flow per beat through the aorta and pulmonary artery $(70 \mathrm{~mL})$ measured with MRI.

\section{DISCUSSION}

A PFO is present in $25-30 \%$ of the population; however, most will remain without haemodynamic consequences under normal conditions [3]. In case of increased Pra, right-to-left shunting through a PFO might occur, leading to hypoxaemia $[4,5]$. We presented two cases of right-to-left shunting through a PFO in the presence of PH secondary to COPD, in which shunting significantly contributed to the hypoxaemia. The PH presumably started shunting through the PFO, leading to hypoxaemia. With hypoxaemia, the oxygen pressure in mixed venous blood also decreased, thereby stimulating hypoxic pulmonary vasoconstriction [6], in turn leading to an increase in Ppa. This led to a further increase in Pra and, thereby, to a larger pressure gradient over the $\mathrm{PFO}$, leading to the vicious circle, as shown in figure 1 . This mechanism also explains the severe desaturation observed at exercise, as cardiac output augmentation induced by exercise will lead to an increase in $P$ pa and, thus, an increase in the right-to-left shunt. Finally, BiPAP might induce an increase of the right-to-left shunt, as was observed in patient $\mathrm{B}$, due to its effect on $P$ pa [7].

In both patients, active vasoconstriction contributed to $\mathrm{PH}$ to a great extent, as was proven by the effects of the vasodilating agent NO. Arterial oxygenation improved with vasodilation, in contrast with large groups of COPD patients [8, 9]. This provides evidence that the right-to-left shunt was the main mechanism of hypoxaemia in our patients. Therefore, it was decided to close the PFO in patient $\mathrm{A}$ as a treatment of the cause of the $\mathrm{PH}$, as outlined in figure 1 .

Because of the inability to close the PFO percutaneously in patient B, a trial with a PDE-5 inhibitor was initiated. Although studies revealed no benefit of PDE-5 inhibitors or, even, worsening of the ventilation-perfusion match in COPD patients [9, 10], we started it based on the beneficial response to inhaled NO.

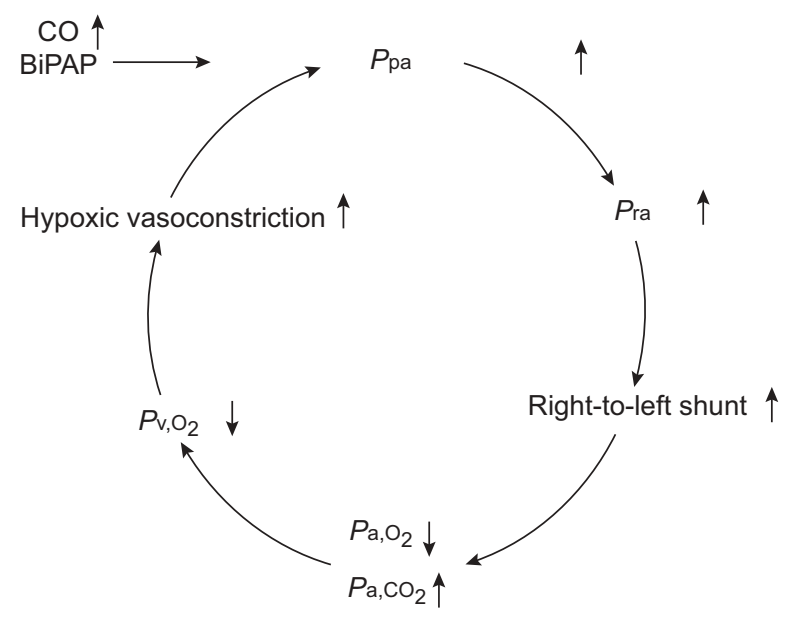

FIGURE 1. Vicious circle of right-to-left shunting in the presence of pulmonary hypertension secondary to chronic obstructive pulmonary disease. $\mathrm{CO}$ : cardiac output; BiPAP: bilevel positive airway pressure; Ppa: pulmonary artery pressure; Pra: right arterial pressure; $\mathrm{Pa}, \mathrm{O}_{2}$ : arterial oxygen tension; $\mathrm{Pa}, \mathrm{CO}_{2}$ : arterial carbon dioxide tension; $P \mathrm{v}_{1} \mathrm{O}_{2}$ : mixed venous oxygen tension.

Our aim was to lower Ppa and Pra, leading to closure of the PFO. Because of a good response and clinical improvement, we decided to continue treatment with the PDE-5 inhibitor. However, no conclusions should be drawn from our observations on how we should treat COPD patients with $\mathrm{PH}$ in general. Both patients were already on oxygen treatment, which was continued afterwards, since oxygen therapy is the only proven beneficial therapy for COPD and $\mathrm{PH}$.

Why was initially the PFO not seen with echocardiography in both patients? Most probably because these measurements were performed at rest with administration of $15 \mathrm{~L} \cdot \mathrm{min}^{-1}$ oxygen. With the vasodilatory effect of oxygen, $P$ ra, the driving force of the right-to-left shunt, was lowered and the PFO was physiologically closed.

Thus, where pulmonary shunting in patients with COPD is usually not a cause of hypoxaemia, right-to-left shunting through a PFO can contribute to the hypoxaemia in presence of secondary $\mathrm{PH}$. The importance of the shunting might be underestimated if echocardiographic evaluation is performed while the patient uses high-fraction oxygen therapy. Traditionally, it is assumed that a trial with a high inspiratory oxygen fraction is a good measure to quantify shunt. Our two cases showed that, in the case of COPD, this rule does not always apply.

\section{B.G. Boerrigter, A. Boonstra, N. Westerhof, P.E. Postmus and}

\section{A. Vonk-Noordegraaf}

Dept of Pulmonary Diseases, VU University Medical Center, Amsterdam, The Netherlands.

Correspondence: A. Vonk-Noordegraaf, Dept of Pulmonary Diseases, VU University Medical Center, De Boelelaan 1117, 1081 HV Amsterdam, The Netherlands. E-mail: a.vonk@vumc.nl

Statement of Interest: A statement of interest for A. Boonstra can be found at www.erj.ersjournals.com/site/misc/statements.xhtml 


\section{REFERENCES}

1 Marthan R, Castaing Y, Manier G, et al. Gas exchange alterations in patients with chronic obstructive lung disease. Chest 1985; 87: 470-475.

2 Wagner PD, Dantzker DR, Dueck R, et al. Ventilation-perfusion inequality in chronic obstructive pulmonary disease. J Clin Invest 1977; 59: 203-216.

3 Hagen PT, Scholz DG, Edwards WD. Incidence and size of patent foramen ovale during the first 10 decades of life: an autopsy study of 965 normal hearts. Mayo Clin Proc 1984; 59: 17-20.

4 Herve P, Petitpretz P, Simonneau G, et al. The mechanisms of abnormal gas exchange in acute massive pulmonary embolism. Am Rev Respir Dis 1983; 128: 1101-1102.

5 Oudiz RJ, Midde R, Hovenesyan A, et al. Usefulness of right-to-left shunting and poor exercise gas exchange for predicting prognosis in patients with pulmonary arterial hypertension. Am J Cardiol 2010; 105: 1186-1191.
6 Marshall C, Marshall B. Site and sensitivity for stimulation of hypoxic pulmonary vasoconstriction. J Appl Physiol 1983; 55: 711-716.

7 Jardin F, Delorme G, Hardy A, et al. Reevaluation of hemodynamic consequences of positive pressure ventilation: emphasis on cyclic right ventricular afterloading by mechanical lung inflation. Anesthesiology 1990; 72: 966-970.

8 Barbera JA, Roger N, Roca J, et al. Worsening of pulmonary gas exchange with nitric oxide inhalation in chronic obstructive pulmonary disease. Lancet 1996; 347: 436-440.

9 Blanco I, Gimeno E, Munoz PA, et al. Hemodynamic and gas exchange effects of sildenafil in patients with chronic obstructive pulmonary disease and pulmonary hypertension. Am J Respir Crit Care Med 2010; 181: 270-278.

10 Rietema H, Holverda S, Bogaard HJ, et al. Sildenafil treatment in COPD does not affect stroke volume or exercise capacity. Eur Respir J 2008; 31: 759-764.

\section{Three colleagues with sarcoidosis?}

\section{To the Editors:}

Bilateral pulmonary nodules are a relatively common finding in thoracic radiology and hence are an important problem that chest physicians often face. Aetiology can be diverse, ranging from neoplastic conditions to infectious lesions. An accurate and prompt diagnosis is needed in order to target the most appropriate and least harmful treatment. We report the case of a 49-yr-old, Caucasian, otherwise healthy male patient with bilateral lung nodules and an unusual diagnosis.

The patient had an unremarkable history and was referred due to asymptomatic bilateral lung nodules. He had stopped smoking 6 months earlier and had a normal chest radiograph a year before referral, taken for follow-up of benign colon polyps. At the time of presentation he was not on any medication.

The patient was an engineer working in a Belgian high-tech facility. His job mainly consisted of office work with occasional visits to production sites where coating of medical material was performed. When visiting these sites, he did not systematically wear a protective mask. The problems occurred after a business trip to the USA where he met with three colleagues. He first visited his general practitioner with a flu-like syndrome. Other symptoms included a productive cough, night sweats and loss of weight (5 kg in 2 weeks). He was treated symptomatically with paracetamol. Two of his colleagues developed the same complaints. One never visited a doctor but the other did and was diagnosed with sarcoidosis based on the finding of non-necrotising granulomatous lesions in transbronchial biopsies. He was put on steroids and his condition improved. Oddly, the third colleague who did not visit the doctor had an existing diagnosis of sarcoidosis but remained asymptomatic.

Due to persistent cough and low-grade fever, our patient was subsequently referred to a pneumologist. Biochemistry revealed an inflammatory syndrome (C-reactive protein $4.6 \mathrm{mg} \cdot \mathrm{dL}^{-1}$, sedimentation rate $28 \mathrm{~mm} \cdot \mathrm{h}^{-1}$ ) and normal tumour markers, angiotensin-converting enzyme (ACE) levels and anti-nuclear factor (ANF). Mycoplasma pneumoniae serology was compatible with a recent infection. Computed tomography (CT) of the chest showed bilateral lung nodules, some with cavitation, in a subpleural and more central distribution. Extensive mediastinal lymphadenopathy was also present. Bronchoscopy revealed a normal bronchial tree. Bronchoalveolar lavage was neutrophilic $(60 \%)$ with no bacterial pathogens and a normal cytology. Pulmonary function tests were normal (vital capacity $4.9 \mathrm{~L}$, forced expiratory volume in $1 \mathrm{~s}$ (FEV1) 4.4 L, FEV1/forced vital capacity (FVC) $88 \%$, total lung capacity $9.2 \mathrm{~L}$, diffusion capacity $99 \%$ ). Atypical pneumonia was diagnosed and the patient was treated with doxycycline $200 \mathrm{mg}$ daily for 10 days.

2 months after initial presentation the symptoms disappeared and biochemistry normalised. The lung nodules persisted but the cavitations and adenopathy regressed. The patient was then referred to our centre for a second opinion. At that time he was completely asymptomatic and the initial weight lost had been regained. Our differential diagnosis included chronic berylliosis, sarcoidosis and, less likely, malignancy and opportunistic infections. A lymphocyte transformation test (LTT) performed for berylliosis was negative. Bronchoscopy with lavage, brushing and biopsies was repeated and showed a normal cell count and differentiation, a CD4/CD8 ratio of 3.8 and no bacterial pathogens on cultures. Bronchial brushing and biopsies were normal. HIV test was negative.

5 months after his business trip the patient remained asymptomatic. No further change of the radiological picture was noted. Exposure to beryllium seemed very unlikely since the LTT was negative. Given the uncertainty of the diagnosis, a lung biopsy was performed. This revealed necrotising granulomas with a positive Grocott methenamine silver (GMS) stain (fig. 1). A serological test for Histoplasma capsulatum antibodies confirmed 